\title{
Continuous Cooling Curve Diagrams of Isotactic-Polypropylene/ Polyethylene Blends: Mutual Nucleating Effects under Fast Cooling Conditions
}

Enrico Carmeli, Gottfried Kandioller, Markus Gahleitner, Alejandro J. Müller,* Davide Tranchida,* and Dario Cavallo*

Cite This: Macromolecules 2021, 54, 4834-4846

Read Online

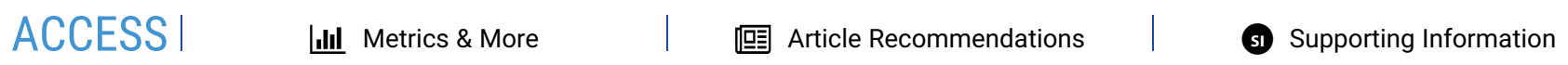

ABSTRACT: Crystallization kinetics under processing-relevant cooling conditions of polyethylene/isotactic-polypropylene ( $\mathrm{PE} / \mathrm{PP})$ blends, with compositions similar to real recycled blends, was investigated through an in situ temperature monitoring technique. Continuous Cooling Curve (CCC) diagrams in a large crystallization temperature range were obtained for the two phases, thanks to a new apparatus allowing fast quenching of polymer samples. Using this technique, we found an "inversion point" in the crystallization order of the two polymers, which arises from the difference in crystallization rates between $\mathrm{PP}$ and $\mathrm{PE}$ with an increasing cooling rate. The order of crystallization of the two polymers at low cooling rates, that is, before the inversion point, can be tuned by employing non-nucleated or nucleated PP. In particular, non-nucleated PP crystallizes after PE

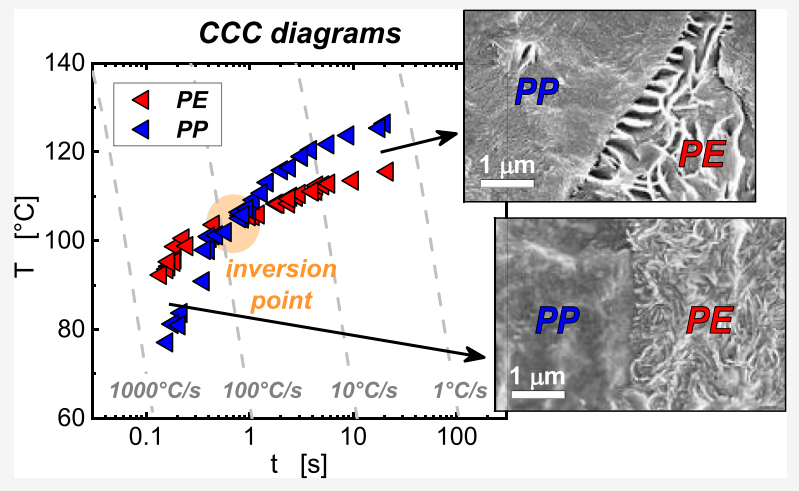
above $0.5{ }^{\circ} \mathrm{C} / \mathrm{s}$, while nucleated PP solidifies before PE until $80^{\circ} \mathrm{C} / \mathrm{s}$. Interestingly, mutual nucleating effects, previously shown only for ideal systems such as thin-layered films, were found at the interface between the phases, in correlation with the inversion point. During cooling, the phase which crystallizes first can enhance the onset of crystallization of the second lower crystallizing phase through nucleation at the interface. Ex situ morphological and wide-angle X-ray diffraction experiments confirmed the validity of the results shown by CCC diagrams. Our approach to characterize semicrystalline polymer blends with CCC diagrams facilitates tailoring their properties at cooling rates relevant for polymer processing. Moreover, the importance of knowing and controlling the type of components in blends obtained from recycling is demonstrated, given the variety of crystallization behaviors attainable.

\section{INTRODUCTION}

Polyolefins are the most produced polymers in terms of tons per year and thus also the most available as feedstock for recycling. Thus, a vast amount of these materials, especially polyethylenes (PEs) and isotactic-polypropylenes (PPs), with very different properties and characteristics, is obtained from recycling. ${ }^{1}$ However, given the similarity of their physical and chemical properties, standard mechanical separation processes are not able to completely divide the two polymer types during sorting of the wastes, resulting in recycled blends. ${ }^{2}$

In addition to the complexity of the composition of recycled blends, PE and PP are immiscible. This phenomenon is due to the small differences in the chemical structure of the macromolecules, disfavoring intermolecular interactions ${ }^{3}$ and leading to multiphasic systems generally characterized by inferior properties as compared to those of the single components. ${ }^{4}$ As such, the understanding and control of structure formation under processing conditions is a key requirement to boost the usage of postconsumer-recycled polyolefin blends.

To unveil the complex crystallization behavior of recycled $\mathrm{PE} / \mathrm{PP}$ blends, the first approach consists of studying crystallization of blends in different compositions, which have been prepared in a laboratory using different virgin materials. Several studies on PE/PP blends were carried out in the last decades to relate together the structure, properties, and processing conditions for academic and industrial purposes. ${ }^{5-13}$ A point of agreement between different authors is that the presence of PE in a blend with PP reduces the nuclei density of the latter. However, the growth rate of $\mathrm{PP}$ was found unchanged in immiscible blends with high-density PE (HDPE) and lowdensity PE (LDPE), ${ }^{3,5,7,9,10}$ while a significative decrease in

Received: March 29, 2021

Revised: April 20, 2021

Published: May 4, 2021

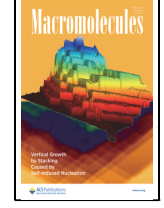


growth rate was found for blends of PP and linear-low-density PE (LLDPE). ${ }^{3}$ In the work of Blackadder et al., ${ }^{12}$ enhancement of the crystallization kinetics of PP blended with HDPE with respect to the neat components was found. On the contrary, Rybnikár et al. ${ }^{10}$ reported a higher crystallization rate of linear PE blended with PP, which was explained as changes in the concentration of nuclei of different types. However, in a more recent work of Rybnikár et al., ${ }^{13}$ the enhancement of the linear PE crystallization rate was attributed to the nucleating action of the previously crystallized PP phase upon cooling from the melt. Nevertheless, a thorough understanding of the crystallization behavior of $\mathrm{PE} / \mathrm{PP}$ blends upon processing-relevant conditions is still lacking.

For achieving an increased output, polymers are always solidified under fast cooling conditions, frequently in combination with high pressure and shear, which significantly affect the final crystallization. ${ }^{14}$ Several studies on the crystallization behavior in a range of cooling rates typical of industrial processes, that is, from a few to thousand ${ }^{\circ} \mathrm{C} / \mathrm{s}$, were published for PP and PE as single components. ${ }^{15-27}$

On the one hand, crystallization of $\mathrm{PP}$ at high cooling rates occurs at extreme undercooling and is often accompanied by major structural changes with the possible development of a metastable phase. Typically, for homopolymer PP, the formation of the stable $\alpha$-phase competes with the development of a "mesophase" in a range of cooling rates between 10 and 100 ${ }^{\circ} \mathrm{C} / \mathrm{s} .{ }^{15,16,23,28}$ The conformation of the PP mesophase consists of $3 / 1$ helices, similar to the other crystalline structures of PP, but the sense of the helices has no particular rule. ${ }^{29}$ Therefore, the degree of order of the PP mesophase is intermediate between that of the amorphous state and that of the monoclinic structure, resulting in a material with peculiar macroscopic properties (e.g., density or elastic modulus). In contrast to this, a different behavior is shown by nucleated PP. Depending on the efficiency of the specific nucleating agent toward PP, the critical cooling rate, above which a predominant fraction of the mesomorphic form is generated, ${ }^{17}$ shifts toward higher values. ${ }^{20-22}$ For very efficient nucleating agents, such as poly(trimethylallylsilane) or poly(vinylcyclohexane) (PVCH), the iPP $\alpha$-phase domain extends to undercoolings at which homogeneous nucleation occurs, giving rise to a nodular morphology, typical of iPP mesophase, rather than a lamellar morphology. ${ }^{30}$

On the other hand, PE generally presents an about 10 times higher crystallization rate than PP and it does not form bulk metastable phases at undercooling relevant to industrial processing conditions. However, as well as for PP, PE crystallization is a highly relevant scientific topic, since the generation of largely different semicrystalline morphologies and, consequently, properties is possible, by, for instance, the variation of the chain architecture (presence of long or short branches) or by the applied thermal history..$^{26,27,31}$ The need of extreme cooling conditions for the study of PE was fulfilled with the development of fast scanning chip calorimetry (FSC), by which the limitations on the maximum achievable cooling rate have been overcome, thanks to a significant reduction of both the sample mass (in the order of tens of nanograms) and the heat capacity of the furnaces. ${ }^{32,33}$ An example of the application of FSC to polyolefin blends is the work of Luijsterburg et al., ${ }^{2}$ in which recycled blends of PE and PP with limited amounts of PE phase as the dispersed component were studied. However, in multiphasic systems, the use of such tiny amount of material (around tens of $\mathrm{pL}$, i.e., $10^{4} \mu \mathrm{m}^{3}$ ) might present problems in terms of homogeneity and nonbulk crystallization behavior of the samples. For example, PE/PP blends have domains that can reach dimensions of up to $50 \mu \mathrm{m}$ or even more in co-continuous systems, and therefore, care should be taken when using the FSC technique to study these kinds of systems.

Thus, given the importance of studying crystallization under processing-relevant cooling conditions, several experimental fast cooling devices have been developed in the last decades. A brief overview on these devices can be found in our previous publication. ${ }^{28}$ However, regarding this topic, no specific studies can be found in the literature on PE/PP blends. Given the immiscibility of $\mathrm{PE}$ and $\mathrm{PP}$, each component will largely crystallize independently upon cooling from the phase-separated melt. ${ }^{34}$ Despite this, some interesting mutual nucleating effects between the two polymers can arise. In particular, epitaxial growth of PE onto polypropylene (and vice versa) has been extensively documented in oriented systems, establishing the contact planes between the two structures. ${ }^{35,36}$ Flow fields in typical polymer processing (e.g., injection molding or extrusion) cause chain stretching and orientation, favoring epitaxy of the PE phase on the oriented PP crystals, when crystallized at higher temperatures. ${ }^{37-40}$ The occurrence of epitaxial crystallization in the flow-oriented specimen significantly improves the interfacial adhesion between the two phases, leading to a remarkable enhancement in both strength and toughness of the material. $^{40-43}$

However, the role of the cooling rate on epitaxial crystallization in $\mathrm{PP} / \mathrm{PE}$ blends is still controversial. ${ }^{38,39}$ Isotropic blends of the two polyolefins exhibit an elastic modulus larger than the weighted average of the pure components when quenched, but lower if cooled slowly. ${ }^{44,45}$ The unusual effect of the cooling rate on the mechanical properties was attributed to the differences in the crystallization temperatures of the neat components but still lacks a detailed experimental investigation under near-processing cooling conditions.

The technique used in this investigation consists of cooling at different rates, while in situ temperature recording is carried out by means of a microthermocouple embedded in the polymeric film, as previously carried out in several studies for single-phase materials. ${ }^{17,19,25,26,46-48}$ In this way, Continuous Cooling Curve (CCC) diagrams can be achieved, which describe the crystallization kinetics through the time-temperature coordinates at which crystallization occurs during cooling. To the best of our knowledge, this technique has never been applied for studying multiphasic systems. In the Experimental Section of this paper, a detailed description of how CCC diagrams for multiphasic systems can be obtained is presented. The studied blends had a composition similar to postconsumer-recycled polyolefin blends and, therefore, contain LDPE, HDPE, and different PP types (nucleated and non-nucleated). However, we limit ourselves to model systems comprising virgin polyolefins only, to provide basic knowledge on the crystallization behavior of the blends. The in situ temperature measurements revealed the presence of mutual nucleating effects between the phases of the blends depending on the applied cooling condition. Morphological and structural characterization was performed to confirm the validity of such findings.

\section{EXPERIMENTAL SECTION}

2.1. Materials. All the materials employed in this investigation were kindly provided by Borealis Polyolefine $\mathrm{GmbH}$. Two types of PP homopolymers were employed, one containing $\mathrm{PVCH}$ as the nucleating agent and the other one containing no nucleating agent. 
These two materials were named PPn and PPp, respectively. An HDPE and an LDPE were also employed. Melt flow rate, molecular weight, and density values for each polymer employed in this investigation can be found in Table 1 . Blends were prepared from these materials by melt

Table 1. Melt Flow Rate, Molecular Weight, and Density Values for Each Employed Material

$\begin{array}{lccc}\text { material } & \text { melt flow rate }{ }^{a}[\mathrm{~g} / 10 \mathrm{~min}] & \mathrm{MW}^{b}[\mathrm{~kg} / \mathrm{mol}] & \text { density }\left[\mathrm{kg} / \mathrm{m}^{3}\right] \\ \text { LDPE } & 0.75 & 122 & 923 \\ \text { HDPE } & 0.30 & 160 & 945 \\ \text { PPn } & 20 & 301 & 900 \\ \text { PPp } & 2.8 & 565 & 900\end{array}$

${ }^{a} \mathrm{PE}$ at $190{ }^{\circ} \mathrm{C}$, PP at $230{ }^{\circ} \mathrm{C}$, load $2.16 \mathrm{~kg}$, ISO $1133 .{ }^{b}$ Hightemperature size exclusion chromatography.

blending at $200{ }^{\circ} \mathrm{C}$ for $10 \mathrm{~min}$ at $100 \mathrm{rpm}$, using a Brabender-type internal mixer. The composition for each blend is reported in Table 2 .

Table 2. Composition of the Prepared Blends

\begin{tabular}{lcccc}
\multicolumn{1}{c}{ blend code } & $\begin{array}{c}\text { LDPE } \\
{[\text { wt \%] }}\end{array}$ & $\begin{array}{c}\text { HDPE } \\
{[\text { wt \%] }}\end{array}$ & $\begin{array}{c}\text { PPn } \\
{[\text { wt \%] }}\end{array}$ & $\begin{array}{c}\text { PPp } \\
{[\text { wt \%] }}\end{array}$ \\
\hline L/H/Pn-20/20/60 & 20 & 20 & 60 & 60 \\
L/H/Pp-20/20/60 & 20 & 20 & & \\
H/Pn-40/60 & & 40 & 60 & \\
L/H-50/50 & 50 & 50 & & \\
\hline
\end{tabular}

Two ternary blends (L/H/Pn-20/20/60 and L/H/Pp-20/20/60) were prepared with a composition typical of postconsumer recycled blends. ${ }^{49}$
The two blends differ only in the type of PP. Furthermore, two binary blends $(\mathrm{H} / \mathrm{Pn}-40 / 60$ and $\mathrm{L} / \mathrm{H}-50 / 50)$ were also prepared to understand the effect of blending with PP and with LDPE on the crystallization behavior of HDPE.

2.2. Methods. 2.2.1. Slow Cooling Experiments with DSC. A TA Instruments DSC 2500, calibrated with indium, zinc, and tin and working under $50 \mathrm{~mL} / \mathrm{min}$ of dry nitrogen, was employed to carry out slow cooling experiments in the range from 3 to $100^{\circ} \mathrm{C} / \mathrm{min}$. For each studied material, a film with a thickness of $200 \mu \mathrm{m}$ was obtained via compression molding. Disks with a diameter of $4 \mathrm{~mm}$ were punched out of such a film and placed in DSC pans. To study the crystallization during cooling from the melt, the sample was first heated at $10^{\circ} \mathrm{C} / \mathrm{min}$ up to $225^{\circ} \mathrm{C}$, held at that temperature for $5 \mathrm{~min}$ to erase the previous thermal history and, then, the cooling scan was recorded at a specific cooling rate down to $20^{\circ} \mathrm{C}$.

To construct CCC diagrams from DSC measurements, a time zero was assigned to the instant at which the temperature was crossing 195 ${ }^{\circ} \mathrm{C}$ during the cooling from $225^{\circ} \mathrm{C}$. Time rescaling is based on the fact that $195^{\circ} \mathrm{C}$ is a fair estimate of the equilibrium melting temperature of the $\alpha$-form of PP. ${ }^{16}$ This consideration was adopted for all the employed materials, since PP is the highest melting component, and this allows the comparison of CCC diagrams among the different materials studied.

2.2.2. Fast Cooling Experiments with a High-Speed Cooling Device. The device employed to perform fast cooling experiments in the range from ca. 1 to $700{ }^{\circ} \mathrm{C} / \mathrm{s}$ was described in detail in a previous paper. ${ }^{28}$ However, a new setup was designed and employed in the present investigation for achieving CCC diagrams. A microthermocouple was used for acquiring the instantaneous temperature of the sample and, therefore, detecting the crystallization phenomena that take place during cooling. The microthermocouple was embedded between two polymer films by interposing it between the two molten a)

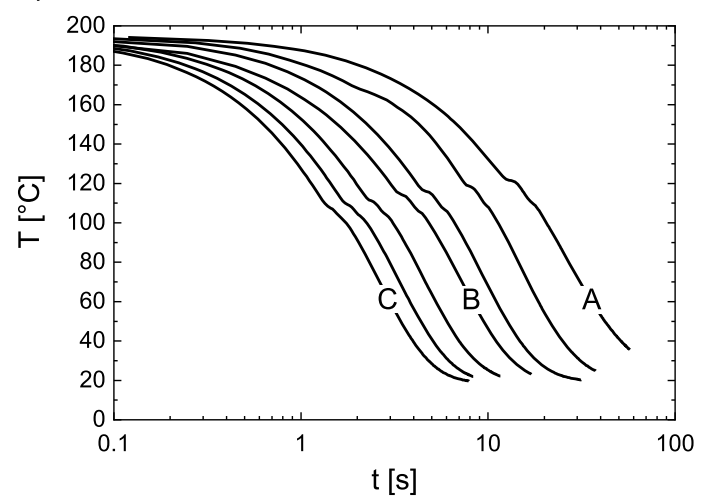

b)

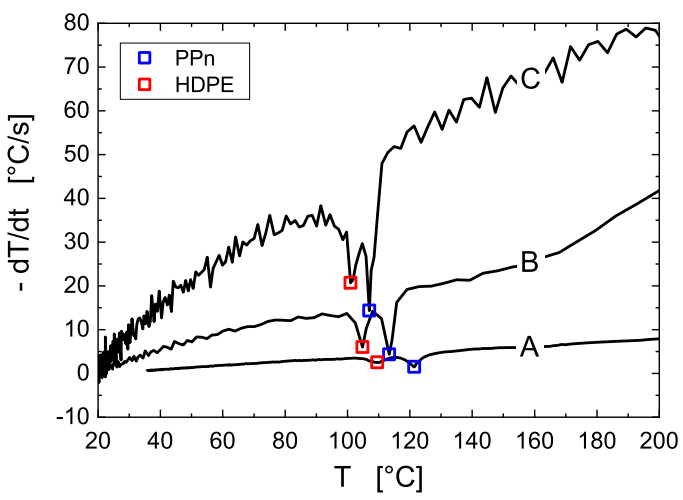

c)

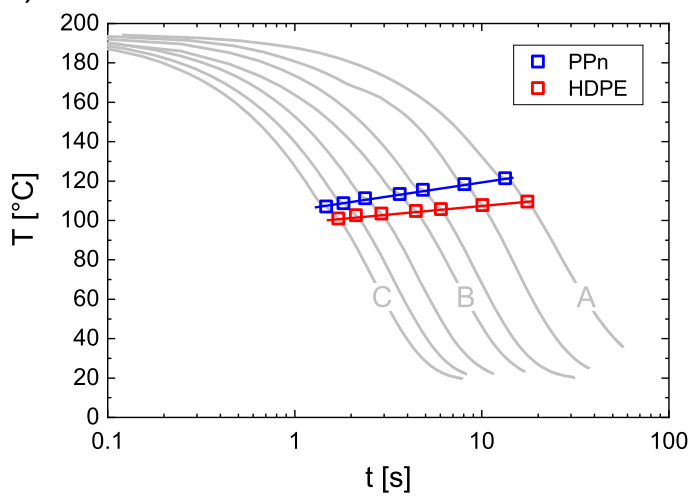

Figure 1. (a) Examples of experimental temperature vs time curves acquired during cooling under different cooling conditions for the $\mathrm{L} / \mathrm{H} / \mathrm{Pn}-20 / 20 /$ 60 blend. (b) Derivative of the temperature with respect to time for curves named A, B, and C in (a). (c) CCC diagram for the PP phase (blue square symbols) and for the PE phase (red square symbols) on top of the experimental curves shown in (a). 
a)

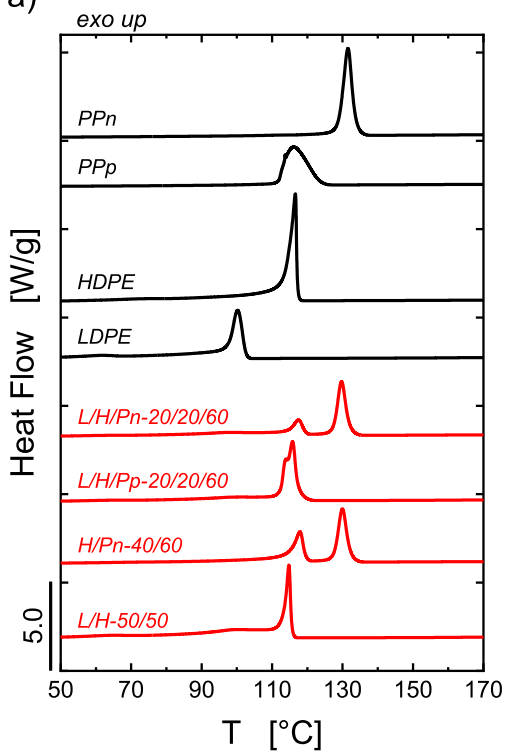

b)

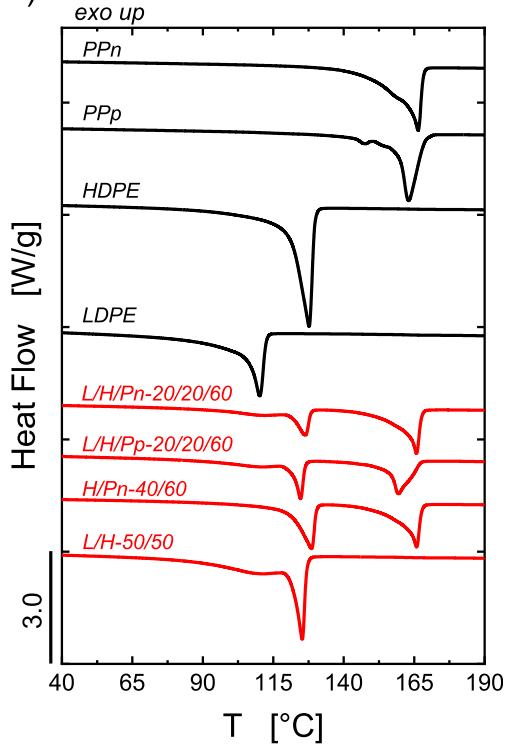

Figure 2. DSC (a) cooling scans and (b) second heating scans at $10{ }^{\circ} \mathrm{C} / \mathrm{min}$ for neat components (black curves) and blends (red curves) studied.

films and by applying slight pressure in order to reach the minimum possible thickness for the final film, that is, about $200 \mu \mathrm{m}$. The obtained film was then wrapped in an aluminum foil to avoid any leakage of the molten material, and the whole package was in turn sandwiched between two slabs fixed to the pneumatic piston. The latter allows moving the sample from the hot zone, where melting of the polymer occurs, to the cold zone, where the cooling experiment is performed. The slabs were made from a metal net to reduce the total mass of the system and to improve the sensitivity of the thermocouple to the phase transitions which occur inside the polymer. A window of ca. $10 \times 10$ $\mathrm{mm}$ was cut out of the slabs, and the aluminum-polymer package was placed in between the slabs within the window. In this way, the cooling medium directly hits the aluminum-polymer package, and the slabs give only a minimum contribution to the heat transfer process. A sketch of the cooling mechanism and the sample holder employed is shown in Figure S1 in the Supporting Information.

Examples of the temperature recorded in time during experiments under different cooling conditions are reported in Figure 1a.

If the thermocouple would not be directly in contact with the polymeric film, upon opening the nozzles, the temperature of the sample would decrease with an exponential-like decay. ${ }^{16,19,28}$ The trend of the temperature decay in time is nonlinear because the cooling effectiveness depends on the difference between the sample and the cooling medium temperature, such that the cooling is more effective at the temperatures where this difference is high, but loses efficiency upon decreasing temperature difference. The consequence is that the cooling experiment is not cooling rate-controlled but rather ballistic. When the crystallization of the material takes place (at different temperatures depending on the cooling conditions and on the crystallization kinetics of the material itself), a deviation from the exponential decay of the temperature in time is observed, as shown in Figure 1a. When the cooling conditions are mild, the rate of heat release, that is, crystallization rate, can be equal or even overcome the rate at which the heat is carried away by the cooling medium, that is, the cooling rate. In the former case, a plateau is observed, while in the latter case, an increase in the temperature during cooling occurs, giving rise to a peak. Upon stronger cooling conditions, however, the rate of release of crystallization heat becomes smaller than the rate of heat transfer toward the cooling medium, and the crystallization process produces a slight deviation from the exponential decay of the temperature in time. In the latter case or in the case of a temperature plateau, the crystallization temperature $\left(T_{c}\right)$ is better obtained from the curve of the derivative of temperature with respect to time $(-\mathrm{d} T / \mathrm{d} t)$ versus temperature as the minimum cooling rate value reached when the deviation from linearity occurs (see Figure $1 \mathrm{~b}$ ).

Finally, by reporting the calculated $T_{c}$ on the experimental temperature versus time plot for each phase, the CCC diagrams are obtained (see Figure 1c).

2.2.3. Scanning Electron Microscopy Analysis. Cross sections of the polymer films, produced during experiments in the High-Speed Cooling Device (HSCD), were obtained via cryo-microtoming at -100 ${ }^{\circ} \mathrm{C}$ (Leica EM UC7). The cryo-cut specimens were etched in a $1 \%$ solution of $\mathrm{KMnO}_{4}$ in $85 \% \mathrm{H}_{2} \mathrm{SO}_{4}$ for $15 \mathrm{~min}$. The specimens were then washed with distilled water, stirred in $30 \% \mathrm{H}_{2} \mathrm{O}_{2}$ solution for $10 \mathrm{~min}$, again washed with distilled water, and finally rinsed with acetone. The produced specimens were sputter-coated with a Pt layer prior to scanning electron microscopy (SEM) analysis. SEM observations were performed on a Thermo Fisher Scientific Apreo S LoVac scanning electron microscope.

2.2.4. Wide-Angle X-ray Scattering Analysis. Wide-angle X-ray scattering (WAXS) measurements were carried out in the reflection mode with a Bruker Discover D8 diffractometer equipped with a twodimensional GADDS detector and Ni-filtered $\mathrm{Cu} \mathrm{K} \alpha$ X-rays.

\section{RESULTS AND DISCUSSION}

3.1. Thermal Properties. In Figure 2, cooling and heating scans obtained in the DSC at $10{ }^{\circ} \mathrm{C} / \mathrm{min}$ are shown for neat and blended components, and the relative values of $T_{\mathrm{c}}$ and $T_{\mathrm{m}}$ are reported in Table 3. The large difference in $T_{c}$ between PPn and $\mathrm{PPp}$ is mainly due to the presence of the highly efficient

Table 3. Thermal Properties of the Materials as Determined From DSC at $10^{\circ} \mathrm{C} / \mathrm{Min}$

\begin{tabular}{lll}
\multicolumn{1}{c}{ material } & \multicolumn{1}{c}{$T_{\mathrm{c}}\left[{ }^{\circ} \mathrm{C}\right]$} & \multicolumn{1}{c}{$T_{\mathrm{m}}\left[{ }^{\circ} \mathrm{C}\right]$} \\
PPn & 131.5 & 166.4 \\
PPp & 116.1 & 163.0 \\
HDPE & 116.6 & 127.7 \\
LDPE & 100.2 & 110.2 \\
L/H/Pn-20/20/60 & $98.6^{a}, 117.5^{b}, 129.7^{c}$ & $112.1^{a}, 126.4^{b}, 165.8^{c}$ \\
L/H/Pp-20/20/60 & $-{ }^{a}, 114.0^{b}, 115.8^{c}$ & $111.3^{a}, 124.7^{b}, 159.4^{c}$ \\
H/Pn-40/60 & $117.9^{b}, 129.9^{c}$ & $128.6^{b}, 165.9^{c}$ \\
L/H-50/50 & $100.4^{a}, 114.8^{b}$ & $111.5^{a}, 125.3^{b}$
\end{tabular}

${ }^{a}$ LDPE. ${ }^{b}$ HDPE. ${ }^{c}$ PP. 
a)

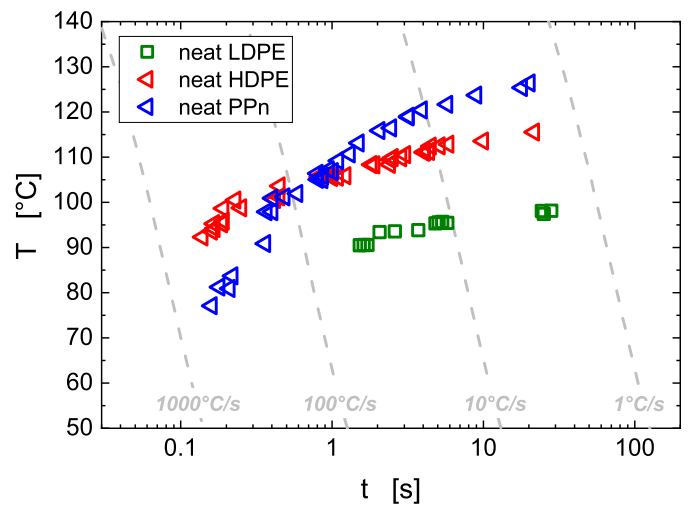

b)

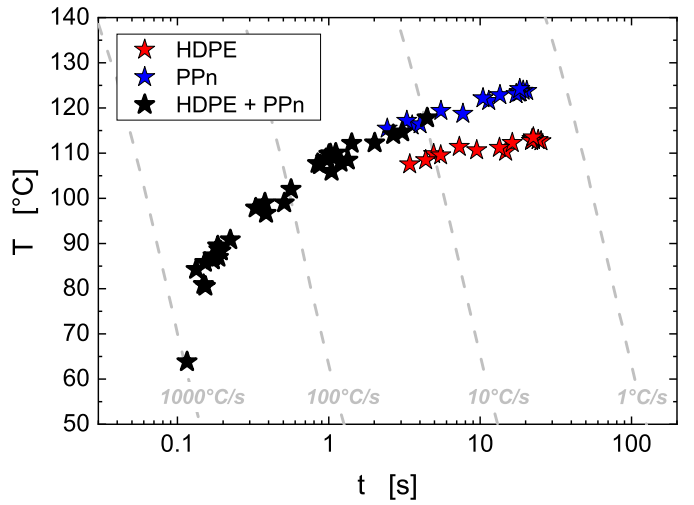

c)

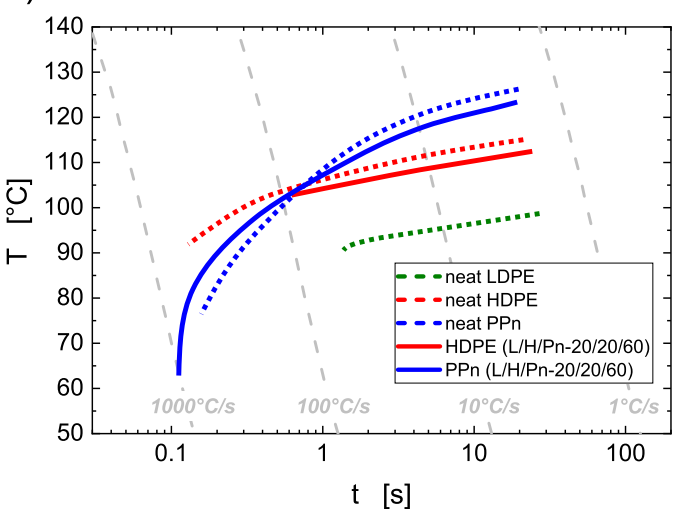

Figure 3. CCC diagrams for (a) neat LDPE, neat HDPE, and neat PPn and for (b) HDPE and PPn as components in the L/H/Pn-20/20/60 blend; data labeled "HDPE + PPn" correspond to concurrent crystallization of PPn and HDPE. (c) CCC diagrams for neat (dashed curves) and blended (solid curves) components (time-temperature coordinates reported in $(\mathrm{a}, \mathrm{b})$ were omitted for the sake of clarity). The gray dashed lines indicate representative ballistic cooling histories from $195{ }^{\circ} \mathrm{C}$ added as examples (cooling rate values calculated at $90{ }^{\circ} \mathrm{C}$ ).

nucleating agent in PPn, which is also responsible for the sharper peak profile. The plot allows comparing the crystallization behavior of the neat components with that of the same components after melt-mixing. Considering the two polypropylenes, $T_{\mathrm{c}}$ after blending slightly decreases for PPp, while it decreases more markedly for PPn. The reason will be discussed in detail later on.

At the same time, for HDPE, the crystallization behavior is more complicated. $T_{\mathrm{c}}$ decreases by approximately $2{ }^{\circ} \mathrm{C}$ after blending with LDPE because of the well-known cocrystallization process, that is, shorter linear segments of LDPE chains and longer crystallizable segments of HDPE chains participate together in the development of crystals. ${ }^{4-54}$ Therefore, for the mixing at the macromolecular scale necessary for the formation of cocrystals to occur, a partial miscibility of the two components must be present in the melt. ${ }^{49}$ The same holds true for the lower $T_{\mathrm{c}}$ of HDPE in the L/H/Pp-20/20/60 blend compared to that in neat HDPE. However, the $T_{\mathrm{c}}$ of HDPE is unexpectedly higher in the two blends containing PPn: HDPE, in the H/Pn-40/60 and in the $\mathrm{L} / \mathrm{H} / \mathrm{Pn}-20 / 20 / 60$ blends, shows an increase of 1.3 and $0.9^{\circ} \mathrm{C}$ as compared to neat HDPE, respectively. Thus, it can be already stated that there is a certain nucleating effect toward HDPE in the presence of PPn domains, while this effect is absent in the presence of PPp domains. A similar effect was already reported by Last ${ }^{55}$ in blends of low-density PE and PP, and by Rybnikar and Kaszonyiova ${ }^{13}$ for binary blends of linear PE and $\mathrm{PP}$ as major and minor components, respectively. Furthermore, in the work of Zhang and Ajji, ${ }^{56}$ a nucleating effect toward the PE phase at the interface in blends of LLDPE and PP was found and related to the difference in $T_{\mathrm{c}}$ between LLDPE and the type of PP used.

Figure $2 \mathrm{~b}$ and Table 3 reveal a decrease in melting temperature $\left(T_{\mathrm{m}}\right)$ for all the components after melt-blending, as observed in our previous investigation for similar systems, ${ }^{49}$ except for LDPE when blended together with HDPE and for $\mathrm{HDPE}$ in the H/Pn-40/60 blend. For LDPE, the reason for such an increase is again due to the formation of joint crystals together with longer linear segments of HDPE, which causes an increase in lamellar thickness and accordingly a higher $T_{\mathrm{m}}$. For HDPE in the $\mathrm{H} / \mathrm{Pn}-40 / 60$ blend, the increase in $T_{\mathrm{m}}$ agrees with the increase in $T_{c}$ detected upon cooling. In the $\mathrm{L} / \mathrm{H} / \mathrm{Pn}-20 / 20 / 60$ blend, $T_{\mathrm{m}}$ of HDPE is higher than the one of HDPE in the $\mathrm{L} / \mathrm{H}$ $50 / 50$ blend, that is, in the absence of PPn, although lower than $T_{\mathrm{m}}$ of neat HDPE, because of the cocrystallization process, which occurs in the presence of LDPE.

Finally, the shoulder to the main melting peak of PPp at about $148{ }^{\circ} \mathrm{C}$ indicates the presence of a certain amount of $\beta$-phase crystals, whose formation is favored at low cooling rates ${ }^{57}$ and in the absence of impurities with nucleating activity. The same PP type in the $\mathrm{L} / \mathrm{H} / \mathrm{Pp}-20 / 20 / 60$ blend shows a smaller low melting shoulder (observable with a higher scale magnification) corresponding to a much smaller amount of $\beta$-phase crystals. The presence of the $\beta$-phase for PPp was confirmed by WAXS 
analysis, and the reason for the differences in $\beta$-phase content between neat and blended PPp will be explained later on.

The complex crystallization behavior of the different components in the blends will be further examined in the following section, in which CCC diagrams obtained at cooling rates between ca. $0.05{ }^{\circ} \mathrm{C} / \mathrm{s}$ (or $3{ }^{\circ} \mathrm{C} / \mathrm{min}$ ) and $700{ }^{\circ} \mathrm{C} / \mathrm{s}$ are shown.

3.2. CCC Diagrams. 3.2.1. Ternary Blend with PPn, HDPE, and LDPE (L/H/Pn-20/20/60). In Figure 3a, the CCC diagrams of neat PPn, neat HDPE, and neat LDPE are shown over a wide range of cooling rates (from ca. 1 to $700{ }^{\circ} \mathrm{C} / \mathrm{s}$ ). The large decrease (about $50{ }^{\circ} \mathrm{C}$ ) of $T_{\mathrm{c}}$ for PPn is in agreement with the results reported by Mileva et al. ${ }^{20}$ on a similar material measured by means of FSC. For HDPE, a decrease in $T_{\mathrm{c}}$ of around $23^{\circ} \mathrm{C}$ was found, similar to the one obtained with the FSC by Zhuravlev et al. for an HDPE grade in the same cooling rate range. $^{27}$ For LDPE, it was not possible to detect the crystallization event at cooling rates higher than ca. $40{ }^{\circ} \mathrm{C} / \mathrm{s}$. The reason is that LDPE crystallizes in a broad temperature range and, thus, the quantity of heat released during crystallization per unit time (or temperature) is too low for the detection limit of the technique. For a more precise comparison, $T_{\mathrm{c}}$ values as a function of ballistic cooling rate for our PPn and our HDPE are reported together with $T_{\mathrm{c}}$ values as a function of constant cooling rate from Mileva et al. ${ }^{20}$ and Zhuravlev et al. ${ }^{27}$ in Figure S2a,b in the Supporting Information, respectively. The cooling rate value in ballistic cooling experiments is selected at a reference temperature at which the maximum growth rate occurs for crystals of the most stable structure for a specific polymer. Therefore, 90 and $75{ }^{\circ} \mathrm{C}$ were chosen for PPn and HDPE, respectively, in line with previous investigations. $^{23,24,26,28,31}$ One should notice that the choice of a different reference temperature for the selection of the cooling rate value will cause a certain shift of the $T_{\mathrm{c}}$ versus cooling rate $(-\mathrm{d} T / \mathrm{d} t)$ curve along the $x$-axis.

The different level of $T_{\mathrm{c}}$ decrease for the two polyolefins is due to their different crystallization kinetics. Indeed, for the same cooling conditions, the rate of crystal formation is higher for PE with respect to $\mathrm{PP}$, for which the presence of the methyl groups and the formation of a helical structure increase the time for achieving a regular arrangement of the crystallizable segments on the growing crystalline front. Still, the ability of PP to form $\alpha$ phase crystals even at such high cooling rates is due to the presence of the highly efficient nucleating agent used, i.e., PVCH. ${ }^{20,28,30,58}$ Indeed, as reported in several previous studies, the PP homopolymer without specific nucleating agents forms mainly the mesophase or remains amorphous at cooling rates above ca. $100{ }^{\circ} \mathrm{C} / \mathrm{s}^{15,16,18,23,24,28}$

For blends of PE and PP, it is noteworthy that since the crystallization peaks obtained upon cooling for the two polyolefins are affected by the cooling rate to different extents, an "inversion point" of the crystallization order should be detected. Therefore, depending on which component crystallizes first upon cooling, an inverted epitaxial growth or nucleating effect can be obtained at the interface between the phases. In other words, either nucleation of PE on the surface of the crystallized PP or vice versa can occur, depending on the employed cooling rate. The existence of similar inversion points has been reported by Mileva et al. concerning the crystallization behavior of heterophasic PP, which is composed of a matrix of PP and rubbery particles made of random ethylene-propylene copolymers. $^{21,22}$ In their system, PE crystals were formed by these copolymer chain segments made of ethylene counits. A noticeable enhancement of the crystallization rate of the PP matrix was detected at high cooling rates, at which PE crystals form before PP crystals. Finally, in the recent work of De Rosa et al. ${ }^{59}$ about crystallization of PP/PE block copolymers, epitaxial crystallization of PE on PP was obtained at low undercooling, that is, when PP was crystallized before PE upon cooling and vice versa at high undercooling.

The CCC diagrams for the L/H/Pn-20/20/60 blend are reported in Figure $3 \mathrm{~b}$. Two separate crystallization events are observed at low cooling rates, with PPn crystallizing before PE during cooling. However, by increasing the cooling rate, the differentiation of PPn and PE crystallization becomes more difficult since the two peaks overlap. Thus, only one crystallization peak is detected, and it is indicated by the black star-shaped symbols. Beyond the inversion point, it is difficult to distinguish the crystallization event of HDPE from the one of $\mathrm{PPn}$, since the amount of PPn in the $\mathrm{L} / \mathrm{H} / \mathrm{Pn}-20 / 20 / 60$ blend is three times higher, and at very high cooling rates, the sensitivity of the technique is reduced to a level at which only the crystallization peak of the main component in the blend can be discerned. Moreover, the PE phase of the $\mathrm{L} / \mathrm{H} / \mathrm{Pn}-20 / 20 / 60$ blend is composed of LDPE and HDPE, but during cooling, only one crystallization peak is recognizable for this mixed $\mathrm{PE}$ phase. This is due to the proximity of the crystallization peaks of LDPE and HDPE, to the cocrystallization phenomenon occurring among them ${ }^{49-54}$ and to the fact that the crystallization event of LDPE is not detectable at cooling rates higher than ca. $40{ }^{\circ} \mathrm{C} / \mathrm{s}$. Therefore, the PE crystallization peak must be attributed to the HDPE component since this highly linear PE gives rise to a sharper and more distinguishable crystallization peak.

In Figure 3c, the CCC diagrams for both neat and blended components for the $\mathrm{L} / \mathrm{H} / \mathrm{Pn}-20 / 20 / 60$ blend are reported for comparison purposes. The data corresponding to overlapped crystallization peaks were considered representative of PPn crystallization, for the reasons addressed before. For the sake of clarity, data corresponding to the time-temperature coordinates of crystallization have been omitted and only the lines that were drawn on top of each data series as guides to the eyes are reported. The same plot reporting only the timetemperature coordinates for neat and blended components can be found in Figure S3 in the Supporting Information.

At low cooling rates, Figure $3 \mathrm{c}$ shows that both neat $\mathrm{PP}$ and neat HDPE show higher $T_{\mathrm{c}}$ with respect to the corresponding blended components. For the PP phase, this finding was already observed from DSC cooling scans from the melt at $10{ }^{\circ} \mathrm{C} / \mathrm{min}$ (see Figure 2a), and it is confirmed by other measurements performed in the whole cooling rate range available with DSC (from 3 to $100{ }^{\circ} \mathrm{C} / \mathrm{min}$ ) (see Figure $\mathrm{S} 4$ in the Supporting Information). Indeed, a constant difference of ca. $1.7^{\circ} \mathrm{C}$ was detected between the $T_{\mathrm{c}}$ of neat and the $T_{\mathrm{c}}$ of blended PPn. In previous studies, this effect of PE on the crystallization kinetics of PP, when melt-blended, was reported and explained as either a diluent effect of the PE phase (still molten at the temperatures at which PP crystallizes) on the crystallizing PP phase $\mathrm{p}^{7,13}$ or as a decrease in the number of heterogeneous nuclei in the PP phase due to their migration toward PE domains. ${ }^{10,60}$ However, the first hypothesis seems not appropriate to explain this phenomenon for the present case study. Indeed, a clear phase separation at the micrometer scale between PE and PP was observed by SEM (see below), as expected from the immiscibility between these two polyolefins. Therefore, a migration of some nucleating impurities from the PP phase 
toward the PE phase seems the only reasonable explanation for the observed decrease in $T_{\mathrm{c}}$ of the PP phase.

From the comparison of the CCC diagrams of blended and neat PPn at very high cooling rates, that is, beyond the inversion point, Figure $3 \mathrm{c}$ shows that blended PPn has a slightly faster crystallization kinetics than neat PPn. This might be due to the presence of a solid interface of PE crystals formed at higher $T_{c}$ which triggers the nucleation of PPn. A verification of this effect was carried out by WAXS. A sample of neat PPn and a sample of the $\mathrm{L} / \mathrm{H} / \mathrm{Pn}-20 / 20 / 60$ blend were placed together in the sample holder and quenched at a high cooling rate (ca. $600{ }^{\circ} \mathrm{C} /$ s) to ensure that the cooling history experienced by the two materials was exactly the same. Then, the corresponding WAXS diffractograms were compared after normalizing the area under the peaks to one and scaling the curve of neat PPn according to the amount of PPn in the L/H/Pn-20/20/60 blend (see Figure 4). The typical crystalline reflections corresponding to the $\alpha$ -

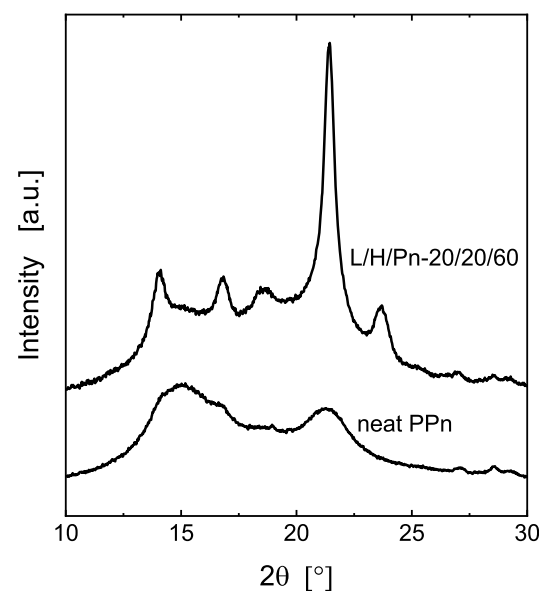

Figure 4. WAXS diffractograms for $\mathrm{L} / \mathrm{H} / \mathrm{Pn}-20 / 20 / 60$ blend and neat $\mathrm{PPn}$ quenched at the same high cooling rate.

phase of PP, that is, at ca. 14,17 , and $18.5^{\circ}$ (due to scattering from the $(110)_{\alpha},(040)_{\alpha}$, and $(130)_{\alpha}$ planes, respectively), are still clearly observable for the PP phase in the L/H/Pn-20/20/ 60 blend. On the contrary, for neat PPn, the $\alpha$-phase peaks have almost completely disappeared, while the two broad peaks generated by the PP mesophase are well developed. This result suggests that in the case of the $\mathrm{L} / \mathrm{H} / \mathrm{Pn}-20 / 20 / 60$ blend, the crystallization rate of PPn is enhanced, and a reasonable explanation for this phenomenon is that, under these cooling conditions, the already formed crystals of PE act as substrates for the formation of PP nuclei as it occurs for heterogeneous nucleation processes.

For the PE phase, the crystallization behavior is more complex. CCC diagrams, obtained by means of DSC measurements at low cooling rates, for the neat HDPE, HDPE in the L/ $\mathrm{H} / \mathrm{Pn}-20 / 20 / 60$, L/H-50/50, and $\mathrm{H} / \mathrm{Pn}-40 / 60$ blend are reported in the same plot in Figure 5a. The CCC diagrams for HDPE in the L/H/Pn-20/20/60 blend and for neat HDPE have nonparallel trends with an increasing cooling rate. A crossing point between them can be detected at a cooling rate of around $30{ }^{\circ} \mathrm{C} / \mathrm{min}$. This means that for lower cooling rates, HDPE in the $\mathrm{L} / \mathrm{H} / \mathrm{Pn}-20 / 20 / 60$ blend has a higher $T_{\mathrm{c}}$ with respect to neat HDPE and vice versa for higher cooling rates, in agreement with the results previously reported in Figures $2 \mathrm{a}$ and $3 \mathrm{c}$. For understanding the reason which causes HDPE in the $\mathrm{L} / \mathrm{H} / \mathrm{Pn}$ $20 / 20 / 60$ blend to have faster kinetics of crystallization with respect to neat HDPE in the very low cooling rate range, CCC diagrams for HDPE in the other two binary blends $(\mathrm{L} / \mathrm{H}-50 / 50$ and $\mathrm{H} / \mathrm{Pn}-40 / 60$ ) should be observed. As expected, when HDPE is blended with LDPE only (L/H-50/50 blend), the cocrystallization process causes its $T_{\mathrm{c}}$ to decrease with respect to neat HDPE by about $1.5{ }^{\circ} \mathrm{C}$ at $3{ }^{\circ} \mathrm{C} / \mathrm{min}$ and by ca. $2.5^{\circ} \mathrm{C}$ at 100 ${ }^{\circ} \mathrm{C} / \mathrm{min}$. Since the PE phase in the $\mathrm{L} / \mathrm{H} / \mathrm{Pn}-20 / 20 / 60$ blend contains the same ratio of LDPE and HDPE as the binary $\mathrm{L} / \mathrm{H}$ $50 / 50$ blend, the higher $T_{\mathrm{c}}$ found for HDPE in the L/H/Pn-20/ 20/60 blend must be due to the presence of PP phase domains, that is, to a nucleating effect at the interface between PE and PP domains, as previously speculated. One should notice the large difference in $T_{\mathrm{c}}$ between $\mathrm{HDPE}$ in the $\mathrm{L} / \mathrm{H} / \mathrm{Pn}-20 / 20 / 60$ blend and HDPE in the $\mathrm{L} / \mathrm{H}-50 / 50$ blend (about $2.8{ }^{\circ} \mathrm{C}$ at $3{ }^{\circ} \mathrm{C} / \mathrm{min}$ and $2.2^{\circ} \mathrm{C}$ at $100{ }^{\circ} \mathrm{C} / \mathrm{min}$ ). This increase in $T_{\mathrm{c}}$ is also found for HDPE in the H/Pn-40/60 blend, for which the crystallization kinetics is even slightly faster than that for HDPE in the $\mathrm{L} / \mathrm{H}$ / Pn-20/20/60, since LDPE is absent and HDPE crystallization is not slowed down by the cocrystallization process.

The results obtained in the low cooling rate range accessible with DSC are all in agreement with the ones achieved at higher cooling rates with the HSCD, which are shown in a narrower temperature range in Figure 5b. The postulated nucleating effect toward PE at the interface with PPn domains also occurs in the higher cooling rate range up to the inversion point of a)

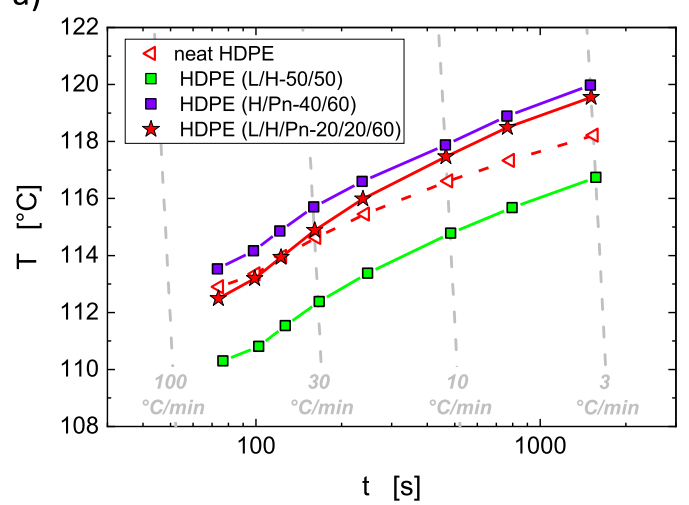

b)

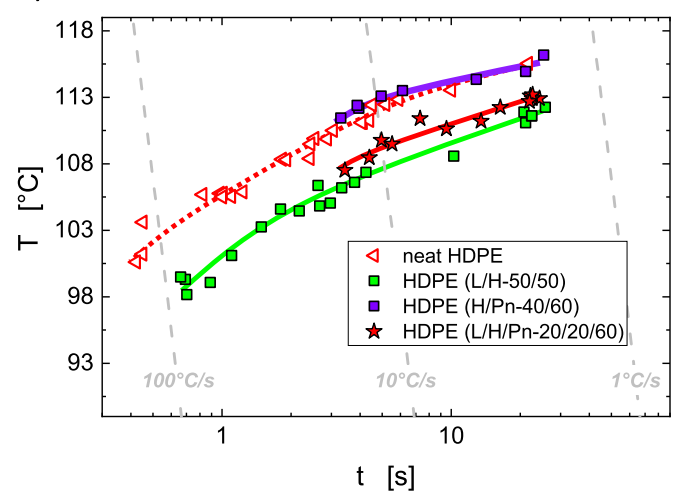

Figure 5. CCC diagrams for neat HDPE and HDPE in the L/H/Pn-20/20/60, L/H-50/50, and H/Pn-40/60 blend obtained with (a) DSC and (b) HSCD. The lines reported for each series are just a guide to the eyes. The gray dashed lines in $(a, b)$ indicate representative constant and ballistic cooling histories from $195^{\circ} \mathrm{C}$ added as example (cooling rate values calculated at $90{ }^{\circ} \mathrm{C}$ ), respectively. 
crystallization. Indeed, in the cooling rate range preceding the inversion point, the $\mathrm{PP}$ phase crystallizes at temperatures above those of HDPE, and, therefore, the HDPE segments at the interface between PP and PE domains may form crystals on top of the previously developed PP crystals.

Finally, the decrease in $T_{c}$ for both PPn and HDPE in the L/ $\mathrm{H} / \mathrm{Pn}-20 / 20 / 60$ blend results in a shift of the inversion point to lower time-temperature coordinates with respect to the two neat components. In terms of cooling rate values at $90{ }^{\circ} \mathrm{C}$ for a ballistic cooling process, the inversion point for the neat components occurs at ca. $60{ }^{\circ} \mathrm{C} / \mathrm{s}$, while for the blended components at ca. $80{ }^{\circ} \mathrm{C} / \mathrm{s}$, which might be a valuable information for relating material properties to different processing conditions.

3.2.2. Ternary Blend with PPp, HDPE, and LDPE $(L / H / P p$ $20 / 20 / 60$ ). In terms of composition, the only difference between the $\mathrm{L} / \mathrm{H} / \mathrm{Pp}-20 / 20 / 60$ and $\mathrm{L} / \mathrm{H} / \mathrm{Pn}-20 / 20 / 60$ blend is the type of PP. The presence of the strong nucleating agent in PPn significantly enhances the formation of its $\alpha$-phase crystals. Indeed, the $T_{\mathrm{c}}$ obtained during cooling at $10^{\circ} \mathrm{C} / \mathrm{min}$ in the DSC for neat PPn is ca. $15{ }^{\circ} \mathrm{C}$ higher than the $T_{\mathrm{c}}$ of neat PPp (see Figures S4 and S5a in the Supporting Information).

To identify the location of the inversion point between neat and blended components of the $\mathrm{L} / \mathrm{H} / \mathrm{Pp}-20 / 20 / 60$ blend, one should look at the CCC diagrams in the DSC cooling rate range (see Figure 6). The inversion point between the neat

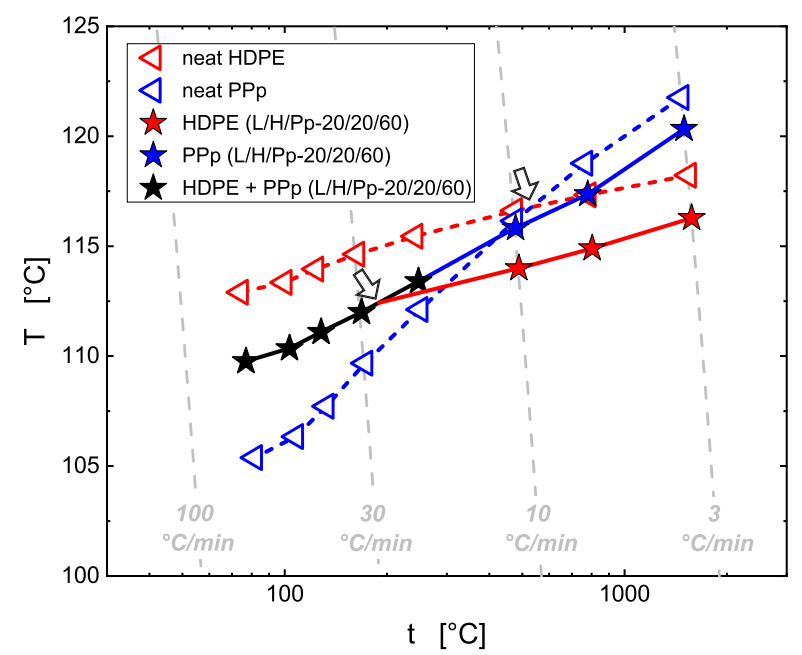

Figure 6. CCC diagrams obtained by means of DSC measurements for $\mathrm{PPp}$ and HDPE as neat and blended components of the $\mathrm{L} / \mathrm{H} / \mathrm{Pp}-20$ / $20 / 60$ blend. The lines connecting each data series are just a guide to the eyes, and the gray dashed lines indicate representative constant cooling histories from $195{ }^{\circ} \mathrm{C}$ added as example. The arrows indicate the inversion point for neat and blended components.

components occurs at about $10{ }^{\circ} \mathrm{C} / \mathrm{min}$. For the blended components, the crystallization peaks are distinguishable from the very low cooling rates up to ca. $20{ }^{\circ} \mathrm{C} / \mathrm{min}$. The data corresponding to overlapped crystallization peaks are reported as black star-shaped symbols. By extrapolating the CCC diagram for the HDPE component, the inversion point can be found to be located at around $20{ }^{\circ} \mathrm{C} / \mathrm{min}$. Therefore, also for the $\mathrm{L} / \mathrm{H} /$ Pp-20/20/60 blend, the inversion point of crystallization between the blended components is shifted to lower timetemperature coordinates as compared to that of the neat components.
In Figure S5a,b in the Supporting Information, the CCC diagrams obtained in DSC for PPp and HDPE, respectively, as neat and blended components are reported for a better comparison of the crystallization behavior before and at the inversion point, that is, in the low cooling rate range. For HDPE, blending with PPp does not produce any enhancement of its crystallization kinetics but rather a slight decrease with respect to HDPE in the L/H-50/50 blend (see Figure S5b in the Supporting Information). Notice that for the $\mathrm{L} / \mathrm{H} / \mathrm{Pn}-20 / 20$ / 60 blend, a substantial increase in $T_{\mathrm{c}}$ was found for the same PE phase when blended with PPn (see Figure 5a). The main reason for the different crystallization behaviors of blended HDPE seems to be the difference in $T_{\mathcal{c}}$, that is, in lamellar thickness, between PPn and PPp. For a clearer comparison of the HDPE crystallization behavior in the two ternary blends studied, the CCC diagrams for HDPE in the L/H/Pn-20/20/60 blend and in the $\mathrm{L} / \mathrm{H} / \mathrm{Pp}-20 / 20 / 60$ blend are reported in the same plot in Figure S6 in the Supporting Information.

From theory, nucleation of a molten phase at the interface with a substrate is known as heterogeneous nucleation, ${ }^{61}$ and depending on the difference among the surface free energies involved in the system, it can enhance the nucleation rate of the crystallizing phase. In case the lattice parameters between the substrate and the overgrowing material match with each other, epitaxial crystallization can occur. In several past studies, the effect of PP on the crystallization behavior of PE has been investigated, resulting in the assessment of epitaxial growth of $\mathrm{PE}$ on PP crystals, due to the dimensional matching of the PE interchain distance $(4.94 \AA)$ with the PP $(10 \overline{1})$ interplanar spacing $(5.05 \AA) .{ }^{35,62-64}$ Furthermore, epitaxial growth occurs when the chain axes of the two phases are about $50^{\circ}$ apart. Thus, the crystal dimension in the substrate must be greater than the critical nucleus size of the depositing phase. As well explained by Greso and Phillips ${ }^{62}$ and experimentally proved by Yan et al., ${ }^{65}$ for the deposition of the first PE stem onto a PP substrate and the generation of a critical nucleus, the critical length of the PE stem must be smaller than the lamellar thickness of the PP substrate in the matching direction.

When comparing the CCC diagrams for neat PPp and neat HDPE obtained in HSCD experiments (see Figure 7), the crystallization of the former occurs at lower temperatures and longer times with respect to the crystallization of the latter, in the whole cooling rate range attainable with HSCD. For the blended components, the crystallization peaks overlap only under the mildest cooling conditions attainable (about $1.5^{\circ} \mathrm{C} / \mathrm{s}$ ), while at higher cooling rates, the crystallization event of HDPE can be separated from the one of PPp, with the former followed by the latter during cooling. Beyond the inversion point, no effects on the crystallization kinetics of HDPE are observable when blended with PPp, since the CCC diagram of HDPE in the L/ $\mathrm{H} / \mathrm{Pp}-20 / 20 / 60$ blend is overlapping with the one of HDPE in the $\mathrm{L} / \mathrm{H}-50 / 50$ blend. In contrast, a strong increase in the crystallization rate of $\mathrm{PPp}$ in the $\mathrm{L} / \mathrm{H} / \mathrm{Pp}-20 / 20 / 60$ blend is observed when compared with that of neat PPp. A difference of about $5{ }^{\circ} \mathrm{C}$ between the $T_{\mathrm{c}}$ of blended and neat PPp at around $1.5^{\circ} \mathrm{C} / \mathrm{s}\left(\mathrm{ca} .100^{\circ} \mathrm{C} / \mathrm{min}\right)$ is in agreement with the DSC results shown in Figure $\mathrm{S} 5 \mathrm{a}$.

To verify the validity of the increased crystallization kinetics for PPp in the L/H/Pp-20/20/60 blend, an ex situ characterization with WAXS of the samples prepared at different cooling rates was performed. In Figure $8 \mathrm{a}, \mathrm{b}$, WAXS diffractograms for neat PPp and the $\mathrm{L} / \mathrm{H} / \mathrm{Pp}-20 / 20 / 60$ blend are shown, respectively. For neat PPp, polymorphism can be observed at 


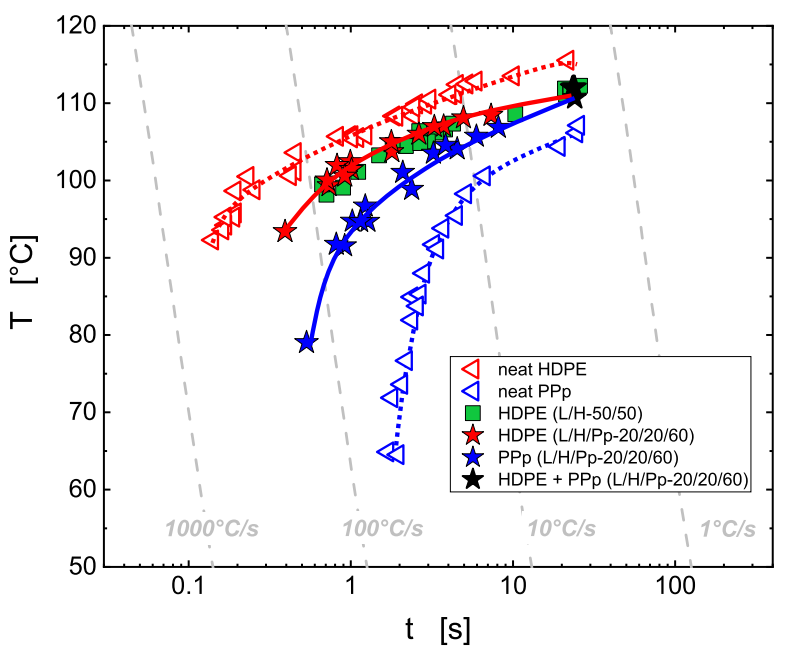

Figure 7. CCC diagrams for HDPE and PPp as neat and blended components of the $\mathrm{L} / \mathrm{H}-50 / 50$ and $\mathrm{L} / \mathrm{H} / \mathrm{Pp}-20 / 20 / 60$ blends. The gray dashed lines indicate representative ballistic cooling histories from $195^{\circ} \mathrm{C}$ added as example (cooling rate values calculated at $90^{\circ} \mathrm{C}$ ). The lines reported for each series are just a guide to the eyes. a)

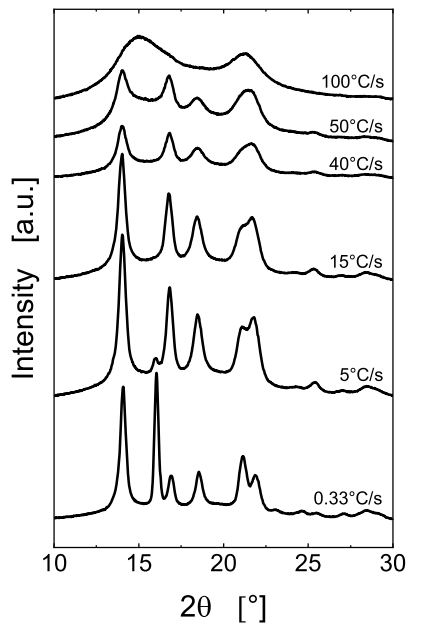

b)

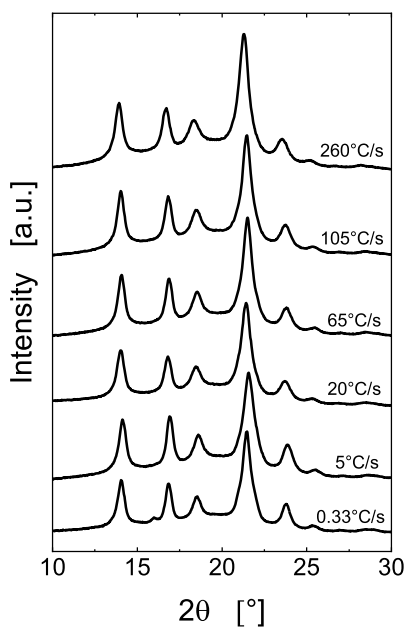

Figure 8. WAXS diffractograms for (a) neat PPp and (b) PPp in the L/ $\mathrm{H} / \mathrm{Pp}-20 / 20 / 60$ blend; each curve is labeled with the cooling rate value representative of the cooling process.

low cooling rates. Indeed, because of the very low amount of heterogeneous particles or residues from the catalyst present in this PP type, $\beta$-crystals can nucleate and grow in large amounts. The characteristic $\beta$-phase peak is located at ca. $16^{\circ}$ in the
WAXS diffractogram. With increasing undercooling, the energy barrier for nucleation is overcome in increasingly more nucleation sites, which usually favors the development of $\alpha$ phase crystals at the expense of $\beta$-phase crystals, because of the faster primary nucleation of the former phase. ${ }^{66}$ Therefore, at 15 ${ }^{\circ} \mathrm{C} / \mathrm{s}$, only $\alpha$-phase characteristic peaks can be observed in the diffractogram. At higher cooling rates, the $\alpha$-to-meso transformation occurs until only a complete mesophase is obtained inside the material at $100{ }^{\circ} \mathrm{C} / \mathrm{s}$. No deconvolution of the WAXS patterns was performed because the diffractograms themselves suffice for the comparison with neat PPp.

For PPp in the L/H/Pp-20/20/60 blend, the contribution of the PE phase to the X-ray pattern of the blend is located at ca. 21.5 and $24^{\circ}$; therefore, the PE peak at a lower angle is overlapped with the two PP peaks located in between 21 and $22^{\circ}$. However, considerations on the polymorphism of PPp can still be made. A much smaller $\beta$-phase peak can be observed at $0.33{ }^{\circ} \mathrm{C} / \mathrm{s}$ in Figure $8 \mathrm{a}$ for the blended PPp as compared to the neat PPp sample cooled at the same rate, and already at $5{ }^{\circ} \mathrm{C} / \mathrm{s}$, only the $\alpha$-phase is detectable. Furthermore, the development of the mesophase, if present, is hardly visible even at $260{ }^{\circ} \mathrm{C} / \mathrm{s}$. These results confirm the increase in the crystallization kinetics of PPp in the $\mathrm{L} / \mathrm{H} / \mathrm{Pp}-20 / 20 / 60$ blend, which can be explained with the same process of heterogeneous nucleation at the interface with $\mathrm{PE}$ domains proposed for the crystallization behavior of PPn in the $\mathrm{L} / \mathrm{H} / \mathrm{Pn}-20 / 20 / 60$ blend beyond the inversion point.

In Figure S7, CCC diagrams for the two neat PPs employed in this investigation and PPp in the $\mathrm{L} / \mathrm{H} / \mathrm{Pp}-20 / 20 / 60$ blend are shown for comparing the efficiency of the observed nucleating effect beyond the inversion point.

3.3. Morphological Analysis. Given the interesting reciprocal effects on the crystallization behavior of the blended components, a morphological analysis via SEM was performed on the $\mathrm{L} / \mathrm{H} / \mathrm{Pn}-20 / 20 / 60$ and $\mathrm{L} / \mathrm{H} / \mathrm{Pp}-20 / 20 / 60$ blends.

The L/H/Pn-20/20/60 blend was prepared under two cooling conditions (see Figure $3 \mathrm{~b}$ ): (i) before the inversion point (at a cooling rate of about $1.5{ }^{\circ} \mathrm{C} / \mathrm{s}$ ), where $\mathrm{PPn}$ crystallization precedes the one of HDPE upon cooling, and (ii) beyond the inversion point (at a cooling rate of about $100^{\circ} \mathrm{C} / \mathrm{s}$ ), where HDPE crystallizes before PPn upon cooling.

In Figure S8 in the Supporting Information, the image of a cross section of the $\mathrm{L} / \mathrm{H} / \mathrm{Pn}-20 / 20 / 60$ blend prepared before the inversion point indicates an interpenetrating continuous structure for both polymers with inclusions of PE within the locally continuous domains of PPn. The assignment of the domains to one phase or the other is based on the composition of the blend, since the PP phase is always the continuous matrix, and on the appearance of the crystalline morphology, with $\mathrm{PE}$
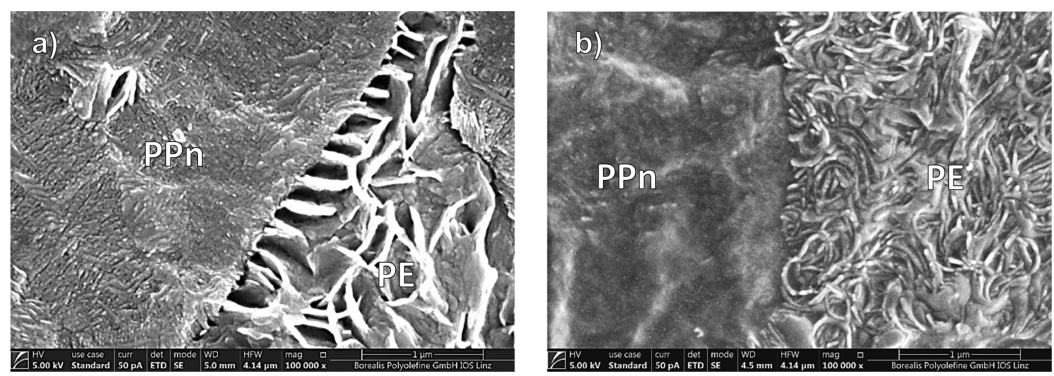

Figure 9. SEM images of the $\mathrm{L} / \mathrm{H} / \mathrm{Pn}-20 / 20 / 60$ blend prepared (a) before the inversion point and (b) beyond the inversion point. PPn and PE domains are indicated on the images. 

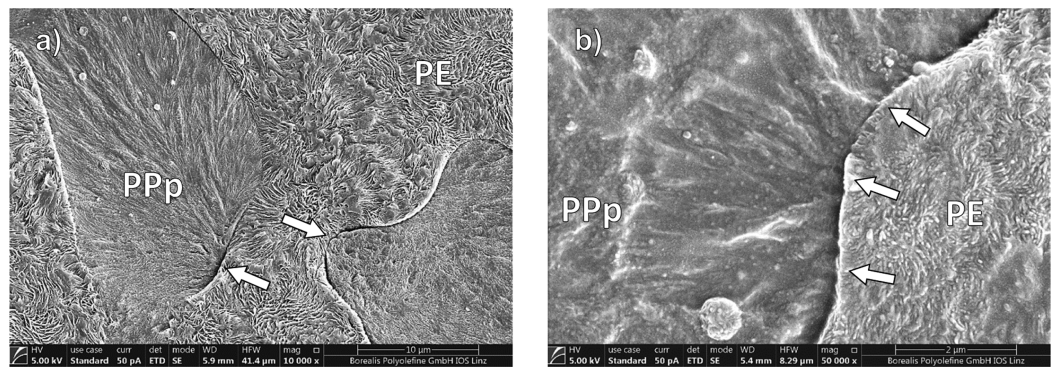

Figure 10. SEM images of the $\mathrm{L} / \mathrm{H} / \mathrm{Pp}-20 / 20 / 60$ blend prepared (a) before the inversion point and (b) beyond the inversion point. PPp and PE domains are indicated on the images, and the arrows indicate the areas where PPp spherulites were most likely nucleated at the interface.

lamellar stacks being thick and easily identifiable, while PP lamellae being thin and arranged in the typical cross-hatched orientation. Noteworthy is the presence in several regions at the interface of PE lamellae oriented perpendicularly to the interface (highlighted by arrows in the image).

An image of the same sample shown in Figure S8 but at higher magnification can be observed in Figure 9a. There is clearly a direct contact between PE and PPn lamellae at the interface, which led to the development of a transcrystalline layer (TCL) of PE onto the already formed PPn crystals. This phenomenon was already shown in some previous investigations, in which a preferential orientation of PE crystals nucleated at the interface with PP domains was reported. ${ }^{12,56}$ However, the phenomenon was not studied under different cooling conditions. In contrast, for the sample prepared beyond the inversion point (Figure 9b), no particular orientation of PE lamellae at the interface is observed, and there seems to be no difference between nucleation density in the bulk and at the interface within the PE domain.

Finally, it was not possible to verify the nucleating effect of $\mathrm{PE}$ on PPn chains beyond the inversion point by means of morphological analysis, since the PP chains crystallize either in mesomorphic nodules with diameters of tens of nanometers or in very thin alpha-phase lamellae, which are not distinguishable in the SEM images. However, WAXS results shown in Figure 4 are a clear confirmation of this phenomenon.

For the L/H/Pp-20/20/60 blend, two samples were prepared: (i) before the inversion point by means of DSC with a cooling rate of $1^{\circ} \mathrm{C} / \mathrm{min}$, at which PPp crystallizes before HDPE (see Figure 6), and (ii) beyond the inversion point by means of $\mathrm{HSCD}$ at ca. $20^{\circ} \mathrm{C} / \mathrm{s}$, at which the crystallization order is inverted (see Figure 7).

In Figure 10a, the image for the sample prepared before the inversion point can be observed. On the one hand, no TCL is detectable for the PE phase at the interface with PPp domains, and there seems to be no difference between nucleation at the interface and in the bulk of PE domains. This result agrees with the absence of any effect on the crystallization behavior of the PE phase in the $\mathrm{L} / \mathrm{H} / \mathrm{Pp}-20 / 20 / 60$ blend, as revealed by CCC diagrams in Figure S5b. On the other hand, PPp spherulites are nucleated at the interface when PE domains are still molten, as indicated by the arrows in the image. This phenomenon is already known in the literature for immiscible blends ${ }^{67}$ and was already observed in SEM images for PE/PP blends. ${ }^{68}$

For the sample prepared beyond the inversion point (see Figure 10b), nucleation sites of PP spherulites are located at the interface as well, but due to the higher undercooling experienced under this cooling condition, the number of available sites for nucleation is much higher, resulting in the impingement of the spherulites. Therefore, this phenomenon causes a noticeable enhancement of the crystallization rate for PPp in the $\mathrm{L} / \mathrm{H} / \mathrm{Pp}$ 20/20/60 blend, as previously described by the CCC diagrams in Figure 7. Furthermore, no preferential orientation of PE lamellae is detectable, in agreement with the absence of any effect on the crystallization rate of the $\mathrm{PE}$ phase predicted by the CCC diagrams in Figure 7. For the sake of clarity, a SEM image at lower magnification and the corresponding sketch representing the nucleation of PPp spherulites at the interface are shown in Figure $S 9$ in the Supporting Information for the sample of the $\mathrm{L} / \mathrm{H} / \mathrm{Pp}-20 / 20 / 60$ blend prepared beyond the inversion point.

\section{CONCLUSIONS}

A custom-made setup able to derive Continuous Cooling Curve diagrams in a wide range of cooling rates (from 1 to $700{ }^{\circ} \mathrm{C} / \mathrm{s}$ ) was employed for the first time to study $\mathrm{PE} / \mathrm{PP}$ immiscible blends. This has allowed us to investigate the crystallization kinetics of the two phases under cooling conditions relevant for processing.

In the blends studied, a clear inversion point of the crystallization order among the phases has been detected by recording Continuous Cooling Curve diagrams due to the different dependences of the crystallization rates of $\mathrm{PP}$ and $\mathrm{PE}$ as cooling rates are increased. The strong dependence of the crystallization behavior of the blends on the type of PP was revealed by the location of inversion points under different cooling conditions. In particular, non-nucleated PP crystallizes after PE above $0.5^{\circ} \mathrm{C} / \mathrm{s}$, while nucleated PP solidifies before PE until $80{ }^{\circ} \mathrm{C} / \mathrm{s}$.

Furthermore, mutual nucleating effects, which have so far only been shown to occur in "ideal" systems only (epitaxy in thinlayered films of PE and PP), were found hereby in bulk blends, and their dependence on the applied cooling condition was studied. A peculiar nucleating effect of a highly nucleated PP toward the lower crystallizing PE phase was detected in the Continuous Cooling Curve diagrams at low cooling rates and confirmed by SEM analysis, where PE lamellae grow perpendicularly to the interface between nucleated PP and PE domains forming transcrystalline layers. As speculated, the transcrystalline layers formed by PE chains at the interface are not anymore present beyond it, where the PE phase crystallizes before the PP phase upon cooling. Moreover, beyond the inversion point, a slight enhancement of the nucleated PP crystallization rate was found and attributed to the nucleation of PP crystals on the already formed PE crystals.

For a second blend with the same composition but containing a homopolymer PP without specific nucleating agents, no effect on the crystallization rate of the PE phase was found, while a noticeable enhancement of the nucleation ability toward nonnucleated PP was detected beyond the inversion point. Indeed, morphological analysis showed a consistent increase in 
nucleation density of PP at the interface for the sample prepared under this cooling condition. The existence or lack of the enhancement of crystallization kinetics of the PE phase under cooling conditions preceding the inversion point was speculated to be an evidence of epitaxial growth of PE crystals onto PP crystals, as its occurrence depends on the difference in crystallization temperature (i.e., lamellar thickness), between the two phases.

The application of the High Speed Cooling Device technique, together with the corresponding structural (WAXS) and morphological (SEM) characterization, provides basic understanding of the solidification of semicrystalline multiphasic polymeric materials under processing-relevant cooling conditions. Such an understanding can be fundamental for the determination of polymeric properties. This study reveals the importance of knowing and controlling the blend composition in the case of real recycled blends of PE and PP, as the type of PP or PE can change dramatically the cooling condition at which the inversion point occurs or can cause an enhanced interaction at the interface between the phases through epitaxial growth.

\section{ASSOCIATED CONTENT}

\section{SI Supporting Information}

The Supporting Information is available free of charge at https://pubs.acs.org/doi/10.1021/acs.macromol.1c00699.

Sketch of the cooling step and sample holder employed for fast cooling experiments; comparison of $T_{c}$ values obtained in FSC from the literature and in HSCD in this work; CCC diagram (experimental data) of neat and blended components of the $\mathrm{L} / \mathrm{H} / \mathrm{Pn}-20 / 20 / 60$ blend; CCC diagrams obtained by means of DSC measurements for neat PPn and PPn in the L/H/Pn-20/20/60 blend; CCC diagram obtained by means of DSC for PPp as neat and blended components in the $\mathrm{L} / \mathrm{H} / \mathrm{Pp}-20 / 20 / 60$ blend and for neat HDPE, HDPE in $\mathrm{L} / \mathrm{H}-50 / 50$, and $\mathrm{L} / \mathrm{H} / \mathrm{Pp}-$ 20/20/60 blends; comparison of CCC diagrams for $\mathrm{HDPE}$ in $\mathrm{L} / \mathrm{H} / \mathrm{Pn}-20 / 20 / 60$ and $\mathrm{L} / \mathrm{H} / \mathrm{Pp}-20 / 20 / 60$ blends; comparison between CCC diagrams of neat PPn, neat PPp, and PPp in L/H/Pp-20/20/60; SEM image of the $\mathrm{L} / \mathrm{H} / \mathrm{Pn}-20 / 20 / 60$ blend prepared before the inversion point; SEM image and the corresponding sketch of the L/H/Pp-20/20/60 blend prepared under a cooling condition after the inversion point (PDF)

\section{AUTHOR INFORMATION}

\section{Corresponding Authors}

Alejandro J. Müller - Polymat and Department of Polymers and Advanced Materials: Physics, Chemistry and Technology, Faculty of Chemistry, University of the Basque Country UPV/ EHU, 20018 Donostia-San Sebastián, Spain; IKERBASQUE, Basque Foundation for Science, 48013 Bilbao, Spain; (1) orcid.org/0000-0001-7009-7715; Email: alejandrojesus.muller@ehu.es

Davide Tranchida - Borealis Polyolefine GmbH, 4021 Linz, Austria; Email: davide.tranchida@borealisgroup.com

Dario Cavallo - Dipartimento di Chimica e Chimica Industriale, Università degli studi di Genova, 16146 Genova, Italy; 이이.org/0000-0002-3274-7067;

Email: dario.cavallo@unige.it

\section{Authors}

Enrico Carmeli - Dipartimento di Chimica e Chimica Industriale, Università degli studi di Genova, 16146 Genova, Italy

Gottfried Kandioller - Borealis Polyolefine GmbH, 4021 Linz, Austria

Markus Gahleitner - Borealis Polyolefine GmbH, 4021 Linz, Austria

Complete contact information is available at:

https://pubs.acs.org/10.1021/acs.macromol.1c00699

\section{Notes}

The authors declare no competing financial interest.

\section{REFERENCES}

(1) Jubinville, D.; et al. A Comprehensive Review on Global Production and Recycling Methods of Polyolefin (PO) Based Products and Their Post-recycling Applications. Sustainable Materials and Technologies 2020, 25, No. e00188.

(2) Luijsterburg, B. J.; et al. Fast cooling of (non)-nucleated virgin and recycled poly (propylenes): Effect of processing conditions on structural and mechanical properties. Thermochimica Acta 2015, 603, 94-102.

(3) Shanks, R. A.; et al. Polypropylene-polyethylene blend morphology controlled by time-temperature-miscibility. Polymer 2000, 41, 2133-2139.

(4) Pracella, M. Crystallization of polymer blends. In Handbook of Polymer Crystallization; Piorkowka, E., Rutledge, G. C., Eds.; John Wiley \& Sons, Inc., 2013, pp 287-326.

(5) Bartczak, Z.; et al. Spherulite nucleation in blends of isotactic polypropylene with high-density polyethylene. Polymer 1986, 27, 537543.

(6) Gałeski, A.; et al. Spherulite nucleation in polypropylene blends with low density polyethylene. Polymer 1984, 25, 1323-1326.

(7) Blom, H. P.; et al. Isothermal and non-isothermal crystallization of PP: effect of annealing and of the addition of HDPE. Polymer 1998, 39, 4011-4022.

(8) Wenig, W. Zur Phasenstruktur von schlagzähem Polypropylen. Appl. Macromol. Chem. Phys. 1978, 74, 147-164.

(9) Wenig, W.; Meyer, K. Investigation of the crystallization behaviour of polypropylene-polyethylene blends by optical microscopy. Colloid Polym. Sci. 1980, 258, 1009-1014.

(10) Rybnikár, F. Crystallization and morphology in blends of isotactic polypropylene and linear polyethylene. J. Macromol. Sci., Part B: Phys. 1988, 27, 125-144.

(11) Galeski, A.; et al. Polypropylene spherulite morphology and growth rate changes in blends with low-density polyethylene. J. Polym. Sci., Polym. Phys. Ed. 1984, 22, 739-747.

(12) Blackadder, D. A.; et al. Characterization of blends of high density polyethylene with isotactic polypropylene. Macromol. Chem. Phys. 1981, 182, 1271-1282.

(13) Rybnikar, F.; Kaszonyiova, M. Epitaxial crystallization of linear polyethylene in blends with Isotactic polypropylene. J. Macromol. Sci., Part B: Phys. 2014, 53, 217-232.

(14) Pople, J. A.; et al. The development of organized structures in polyethylene crystallized from a sheared melt, analyzed by WAXS and TEM. Polymer 1999, 40, 2769-2777.

(15) Brucato, V.; et al. An experimental methodology to study polymer crystallization under processing conditions. The influence of high cooling rates. Chem. Eng. Sci. 2002, 57, 4129-4143.

(16) Cavallo, D.; et al. Continuous cooling curves diagrams of propene/ethylene random copolymers. The role of ethylene counits in mesophase development. Macromolecules 2010, 43, 2890-2896.

(17) Cavallo, D.; et al. Real-time WAXD detection of mesophase development during quenching of propene/ethylene copolymers. Macromolecules 2010, 43, 10208-10212. 
(18) De Santis, F.; et al. Scanning nanocalorimetry at high cooling rate of isotactic polypropylene. Macromolecules 2006, 39, 2562-2567.

(19) Ding, Z.; Spruiell, J. E. An experimental method for studying nonisothermal crystallization of polymers at very high cooling rates. $J$. Polym. Sci., Part B: Polym. Phys. 1996, 34, 2783-2804.

(20) Mileva, D.; et al. Effect of supercooling on crystal structure of nucleated isotactic polypropylene. Thermochimica Acta 2019, 677, 194-197.

(21) Mileva, D.; et al. Crystallization behaviour of heterophasic propylene-ethylene copolymer at rapid cooling conditions. Polymer 2016, 102, 214-220.

(22) Mileva, D.; et al. New Insights into Crystallization of Heterophasic Isotactic Polypropylene by Fast Scanning Chip Calorimetry. Polymers 2020, 12, 1683.

(23) Piccarolo, S. Morphological changes in isotactic polypropylene as a function of cooling rate. J. Macromol. Sci., Part B: Phys. 1992, 31, 501511.

(24) Piccarolo, S.; et al. Crystallization of polymer melts under fast cooling. II. High-purity iPP. J. Appl. Polym. Sci. 1992, 46, 625-634.

(25) Wagner, J.; et al. Crystallization of ethylene-octene copolymers at high cooling rates. Polymer 1999, 40, 4717-4721.

(26) Wagner, J.; Phillips, P. J. The mechanism of crystallization of linear polyethylene, and its copolymers with octene, over a wide range of supercoolings. Polymer 2001, 42, 8999-9013.

(27) Zhuravlev, E.; et al. Crystallization of polyethylene at large undercooling. ACS Macro Lett. 2016, 5, 365-370.

(28) Carmeli, E.; et al. Instrument for mimicking fast cooling conditions of polymers: design and case studies on polypropylene. Polym. Test. 2021, 97, 107164.

(29) Konishi, T.; et al. Crystallization of isotactic polypropylene from prequenched mesomorphic phase. Macromolecules 2006, 39, 80358040.

(30) De Rosa, C.; et al. Tailoring the properties of polypropylene in the polymerization reactor using polymeric nucleating agents as prepolymers on the Ziegler-Natta catalyst granule. Polym. Chem. 2017, 8, 655-660.

(31) Patki, R. P.; Phillips, P. J. Crystallization kinetics of linear polyethylene: The maximum in crystal growth rate-temperature dependence. European Polymer Journal 2008, 44, 534-541.

(32) Adamovsky, S.; Schick, C. Ultra-fast isothermal calorimetry using thin film sensors. Thermochimica Acta 2004, 415, 1-7.

(33) Adamovsky, S. A.; et al. Scanning microcalorimetry at high cooling rate. Thermochimica Acta 2003, 403, 55-63.

(34) Teh, J. W.; et al. A review of polyethylene-polypropylene blends and their compatibilization. Adv. Polym. Technol. 1994, 13, 1-23.

(35) Lotz, B.; Wittmann, J. C. Polyethylene-isotactic polypropylene epitaxy: Analysis of the diffraction patterns of oriented biphasic blends. J. Polym. Sci., Part B: Polym. Phys. 1987, 25, 1079-1087.

(36) Petermann, J.; Xu, Y. The origin of heteroepitaxy in the system of uniaxially oriented isotactic polypropylene and polyethylene. J. Mater. Sci. 1991, 26, 1211-1215.

(37) $\mathrm{Su}, \mathrm{R}$; et al. Flow-induced epitaxial growth of high density polyethylene in its blends with low crystallizable polypropylene copolymer. Polymer 2011, 52, 3655-3660.

(38) Deng, P.; et al. Epitaxial growth and morphological characteristics of isotactic polypropylene/polyethylene blends: Scale effect and mold temperature. Polym. Test. 2014, 34, 192-201.

(39) Na, B.; et al. Epitaxy growth and directed crystallization of highdensity polyethylene in the oriented blends with isotactic polypropylene. Polymer 2005, 46, 5258-5267.

(40) Niu, B.; et al. Crystallization of linear low density polyethylene on an in situ oriented isotactic polypropylene substrate manipulated by an extensional flow field. CrystEngComm 2016, 18, 77-91.

(41) Chaffin, K. A.; et al. Semicrystalline blends of polyethylene and isotactic polypropylene: Improving mechanical performance by enhancing the interfacial structure. J. Polym. Sci. B Polym. Phys. 2000, $38,108-121$.
(42) Jordan, A. M.; et al. Role of crystallization on polyolefin interfaces: an improved outlook for polyolefin blends. Macromolecules 2018, 51, 2506-2516.

(43) van Drongelen, M.; et al. Flow-induced solidification of highimpact polypropylene copolymer compositions: Morphological and mechanical effects. J. Appl. Polym. Sci. 2015, 132, 42040.

(44) Finlay, J.; et al. Unexpectedly high Young's moduli recorded for iPP/HDPE blends. J. Polym. Sci., Part B: Polym. Phys 2001, 39, 14041414.

(45) Finlay, J.; et al. Mechanical properties and characterization of slowly cooled isotactic polypropylene/high-density polyethylene blends. J. Polym. Sci., Part B: Polym. Phys. 2003, 41, 1384-1392.

(46) Cavallo, D.; et al. Effect of cooling rate on the crystal/mesophase polymorphism of polyamide 6. Colloid Polym. Sci. 2011, 289, 10731079.

(47) Mileva, D.; et al. In situ X-ray analysis of mesophase formation in random copolymers of propylene and 1-butene. Polym. Bull. 2011, 67, 497-510.

(48) Van Erp, T. B.; et al. Rate-, temperature-, and structuredependent yield kinetics of isotactic polypropylene. J. Polym. Sci., Part B: Polym. Phys. 2012, 50, 1438-1451.

(49) Carmeli, E.; et al. A tailor-made Successive Self-nucleation and Annealing protocol for the characterization of recycled polyolefin blends. Polymer 2020, 203, 122791.

(50) Minick, J.; et al. Morphology of HDPE/LDPE blends with different thermal histories. Polymer 1995, 36, 1923-1932.

(51) Morgan, R. L.; et al. Morphology, melting behaviour and cocrystallization in polyethylene blends: the effect of cooling rate on two homogeneously mixed blends. Polymer 1999, 40, 337-348.

(52) Puig, C. C. On the cocrystallization phenomenon in blends of high density polyethylene and low density polyethylene. Polymer Bulletin 1997, 38, 715-720.

(53) Puig, C. C.; et al. Absence of isothermal thickening for a blend of linear and branched polyethylene. Polymer 1993, 34, 3402-3407.

(54) Wignall, G. D.; et al. Morphology of blends of linear and longchain-branched polyethylenes in the solid state: a study by SANS, SAXS, and DSC. Macromolecules 1995, 28, 3156-3167.

(55) Last, A. G. M. Nucleation effects in high polymers. J. Polym. Sci. 1959, 39, 543-545.

(56) Zhang, X. M.; Ajji, A. Oriented structure of PP/LLDPE multilayer and blends films. Polymer 2005, 46, 3385-3393.

(57) Doshev, P.; et al. Phase interactions and structure evolution of heterophasic ethylene-propylene copolymers as a function of system composition. J. Appl. Polym. Sci. 2006, 101, 2825-2837.

(58) Alcazar, D.; et al. Structural matching between the polymeric nucleating agent isotactic poly (vinylcyclohexane) and isotactic polypropylene. Macromolecules 2006, 39, 2832-2840.

(59) De Rosa, C.; et al. Morphology of Isotactic PolypropylenePolyethylene Block Copolymers Driven by Controlled Crystallization. Macromolecules 2020, 53, 10234-10244.

(60) Bartczak, Z.; Gałeski, A. Changes in interface shape during crystallization in two-component polymer systems. Polymer 1986, 27, 544-548.

(61) Mandelkern, L. Crystallization of Polymers: Volume 2, Kinetics and Mechanisms, 2nd ed.; Cambridge University Press, 2004, Vol. 2, pp 67101.

(62) Greso, A. J.; Phillips, P. J. The role of secondary nucleation in epitaxial growth: the template model. Polymer 1994, 35, 3373-3376.

(63) Lotz, B.; Wittmann, J. C. Structural relationships in blends of isotactic polypropylene and polymers with aliphatic sequences. J. Polym. Sci., Part B: Polym. Phys. 1986, 24, 1559-1575.

(64) Yan, S.; et al. Controlling factors for the occurrence of heteroepitaxy of polyethylene on highly oriented isotactic polypropylene. Polymer 1998, 39, 4569-4578.

(65) Yan, S.; et al. Effect of lamellar thickness on the epitaxial crystallization of PE on oriented iPP films. Polym. Bull. 1997, 38, 8794. 
(66) Mollova, A.; et al. Crystallization of isotactic polypropylene containing beta-phase nucleating agent at rapid cooling. Eur. Polym. J. 2013, 49, 1057-1065.

(67) Fenni, S. E.; et al. Nucleation of Poly (lactide) Partially Wet Droplets in Ternary Blends with Poly (butylene succinate) and Poly ( $\varepsilon$ caprolactone). Macromolecules 2020, 53, 1726-1735.

(68) Olley, R. H.; et al. A permanganic etchant for polyolefines. J. Polym. Sci., Polym. Phys. Ed. 1979, 17, 627-643. 